\title{
Texture Perception and Afferent Coding Distorted by Cooling the Human Ulnar Nerve
}

\author{
J. R. Phillips and P. B. C. Matthews \\ The University Laboratory of Physiology, Oxford, OX13PT, United Kingdom
}

\begin{abstract}
The roughness of standacdized surtaces (embassed dot arrays or gratings) was compared by scanning them with the little finger of either hand while the ulnar nerve was cooled unilaterally at the elbow; both hands remained warm. Acrosshand comparison of raughness showed that a given surface felt smoother on the cooled side. When the surfaces were adjusted to foel equally rough, that on the cooled side would narmally have felt appreciably rougher. The effect of the nerve cooling on axonal conduction was monitored during the psychophysical experiments by stimulating the ulnar nerve above the cooled region and recording the EMG of an ulnar-innervated hand muscle. During cooling, large myelinated axons remained unblocked, but prolongation of their absolute retcactory period to 5-10 msec left them unable to transmit trains of impulses at high frequencies (Wedensky inhibition). By varying the length of nerve cooled and the cooling temperature, it was shown that the perceptual effects were not due to an increase in the normal temporal dispersion of impulses transmitted by different-sized afferents. The effect of increasing the absolute refractory period on the signals from the various types of cutaneous afferent was modeled mathematically, using earlier human singlefiber responses to dot arrays. It is concluded that the reduction of perceived roughness with nerve cooling is due to Wedensky inhibition, and that the percept of roughness is related to the local contrast in the afferent spatiotemporal image.
\end{abstract}

[Kay words: somatosensory system, tactile, touch, mechanoreceptors, dispersion, refractory period]

Sensory information from the hand is transmitted to the CNS as patterns of action potentials in arrays of afferent fibers. This essentially simple input is processed centrally to produce a rich perceptual experience, which goes far beyond a simple one-toonc correspondence between the activation of a particular receptor and an awareness of a particular sensation. Individual aspects of perception may be linked to particular features of the peripheral neural code by correlating electrophysiological and psychophysical measurements. The firing patterns evoked by complex cutaneous stimuli, whether in monkey or human, are examined quantitatively to see which features match those of a particular percept (e.g., Mountcastle et al., 1972). In recent such experiments exploring the coding of texture it was concluded

\footnotetext{
Received Oct. 6, 1992; accepted Nov. 25, 1992.

J.R.P. was supported by Wellcome Trust Grant 17733/1.27

Correspondence should be addressed to Dr. John R. Phillips, University Laboratory of Physiology. Parks Road, Oxford, OX1 3PT. UK.

Copyright (C) 1993 Society for Neuroscience $0270-6474 / 93 / 132332-10 \$ 05.00 / 0$
}

that the roughness of a surface could not be coded simply by the mean discharge frequency of cutaneous afferents, but was correlated with certain measures of the variation in their firing rate (Connor et al., 1990). The present article uses a novel method of investigating the peripheral coding of texture, namely, cooling the afferent pathway in humans so as to distort the sensory message and then measuring the resultant changes in sensation (Matthews and Phillips, 1990).

Study of the sensory effects of interfering with nervous conduction by cooling has an honorable history, described by Sinclair and Hinshaw (1951). Bickford (1939), for example, noted that with severe cooling of the ulnar nerve at the elbow the sensation of cold disappears well before that of warmth (coolant down to $-5^{\circ} \mathrm{C}$ ). Such changes in sensory quality have usually been attributed to the selective inactivation of a particular type of afferent fiber. In the present experiments the cooling was deliberately less extreme (coolant down to $8^{\circ} \mathrm{C}$ ) so as to avoid complete block of any type of afferent fiber. However, the sensory discharges were subject to Wedensky inhibition, a classical phenomenon in which high-frequency neural discharges are blocked while low-frequency ones are transmitted (Fulton, 1955), due to an increase in the absolute refractory period of nerve. The occurrence of Wedensky inhibition on cooling is well documented in animal work, with its potential to modify neural signals (Douglas and Malcolm, 1955; Paintal, 1965b; Franz and Iggo, 1968).

In the present experiments, cooling the ulnar nerve at the elbow was found to reduce the perceived roughness of a given surface although the hand remained warm. We chiefly studied surfaces consisting of embossed dots of constant size but variable spacing. These had the particular advantage that they were used in a recent single-unit study in human (Phillips et al., 1992), enabling us to model the changes in spatiotemporal patterning of the afferent discharges produced by cooling. Using the earlier spike trains, the action of cooling was simulated by increasing the absolute refractory period (ARP) to that found during cooling and eliminating those spikes in the normal train that fell within the now prolonged ARP of a previous spike. Simulated cooling compressed the modulation of the neural signals and decreased the contrast in the sensory image; indices of shortterm temporal variation and of local spatial variation in the neural representation of the stimulus were both reduced by an appropriate amount. The hypothesis of Connor et al. (1990) that roughness is coded in terms of the spatiotemporal variation of the afferent responses thus passed a novel test.

\section{Materials and Methods}

Experiments were conducted on five men aged 21-63 years; one of the authors (J.R.P.) was studied in particular detail while developing the 
procedures and was cooled 17 times. Figure 1 shows the essentials of the methods. The nerve cooling was approved by a local ethics committee.

Cooling. Subjects sat with both forearms resting comfortably on a table with the palms of the hands facing downward. The ulnar nerve was cooled unilaterally by circulating chilled water at $8-10^{\circ} \mathrm{C}$ through tubing wrapped around one arm, the other being left free (see Matthews, 1989). The cooling was monitored by a thermocouple placed between the skin and tubing at the elbow; an equilibrium temperature of 10$12^{\circ} \mathrm{C}$ was reached after about 45 min of cooling. Normally the cooling was restricted to about $10 \mathrm{~cm}$ of arm at the elbow; in some experiments $35-40 \mathrm{~cm}$ of arm was cooled, including the elbow. The hand was maintained at about $33^{\circ} \mathrm{C}$ by radiant heat, as measured by a thermocouple on the palm.

Testing roughness perception. Embossed stimulus patterns, concealed from the subject's view, were held stationary on the table and subjects actively scanned them with the pad of the ulnar-innervated little finger on each side. A range of test surfaces were presented to the cooled side for comparison with a standard surface on the unaffected side. On each trial the subject scanned the standard and a particular test surface in succession; they scanned fairly rapidly (approximately $2 \mathrm{~cm}$ strokes at 2-3 Hz), with a force of their own choosing. They then reported whether the test surface felt rougher or smoother than the standard. The response same was not permitted. Typically, six test surfaces (presented 10 times each in pseudorandom order) were used to establish which test surface was equivalent in roughness to the standard. At the beginning of each experiment, a control series of comparisons was made with the tubing in place and with water at $35-38^{\circ} \mathrm{C}$ passing through it; control observations were also normally made after rewarming.

Stimulus surfaces. The usual stimulus surfaces were $50 \times 40 \mathrm{~mm}$ arrays of embossed dots arranged in a tetragonal pattern. Each dot had the form of a truncated cone, $0.43 \mathrm{~mm}$ high and $0.6 \mathrm{~mm}$ wide at its top. The spacing of the dots was $2.0 \mathrm{~mm}$ for the standard surface and increased in $0.1 \mathrm{~mm}$ steps from 1.4 to $3.0 \mathrm{~mm}$ for the various test surfaces. Arrays of dots of different height (range, $0.2-0.67 \mathrm{~mm}$ ) were also available with $2 \mathrm{~mm}$ spacing; however, their profile varied with height, because each set had to be produced independently, rather than all on the same embossed sheet. Experiments were also performed with embossed gratings having a constant bar height $(0.43 \mathrm{~mm})$ and spatial period $(2.0 \mathrm{~mm})$ but with bar widths that increased in $0.1 \mathrm{~mm}$ steps from $0.4 \mathrm{~mm}$ to $1.4 \mathrm{~mm}$. The embossed surfaces were produced commercially by exposing a photosensitive nylon polymer (BASF alcoholwashable nyloprint) to ultraviolet light through a mask consisting of a photographic negative of the pattern. The original patterns were produced using POSTSCRIPT software (Adobe Systems Inc.) and printed on a high-resolution laser printer (1300 dpi or about $20 \mu \mathrm{m}$ resolution).

Monitoring nerve conduction. The effect of cooling on axonal conduction in the ulnar nerve was monitored by stimulating it in the axilla and recording the EMG of the (ulnar-innervated) first dorsal interosseous muscle (FDI) using surface electrodes. Stimuli were delivered via an isolating transformer $(0.4 \mathrm{msec}$ square-wave pulses up to $100 \mathrm{~V})$, and were set to be 10-20\% supramaximal for the EMG. The refractoriness of the cooled nerve was tested by delivering pairs of stimuli at intervals of 5-30 msec. When cooling a long length of nerve, it was more convenient to stimulate the ulnar nerve at the wrist and record the resulting F-wave in FDI to determine the slowing of conduction produced by the cooling (Matthews, 1989), but the refractory period could not then be studied.

Single-unit data used for modeling. The effect on sensory signaling of prolonging the refractory period was modeled using earlier human microneurographic data of the normal discharges of cutaneous mechanoreceptors (Phillips et al., 1992). The data were contained in computer files detailing the responses of 51 single units to repeatedly scanning a dot array across their receptive field. The scans were all in the same direction, but between each scan the dot array was shifted $0.2 \mathrm{~mm}$ at right angles to the scanning direction. The individual embossed dots (dot height, $0.5 \mathrm{~mm}$; dot width, $0.7 \mathrm{~mm}$ ) were similar to those used currently; they were arranged tetragonally in a long strip $(212 \times 25 \mathrm{~mm})$ in which their spacing was constant transversely but decreased linearly from $6.4 \mathrm{~mm}$ to $0.87 \mathrm{~mm}$. The strip was scanned longitudinally at velocities of $60-70 \mathrm{~mm} / \mathrm{sec}$. We analyzed the unitary responses to 10 $\mathrm{mm}$-long sections of the array with mean dot spacings of 2.0 and 2.6 $\mathrm{mm}$, corresponding to the spacings of particular current interest. Dot spacing varied slightly along the regions analyzed, but was always within $5 \%$ of the mean. The relevant trains of action potentials were displayed test surfaces standard

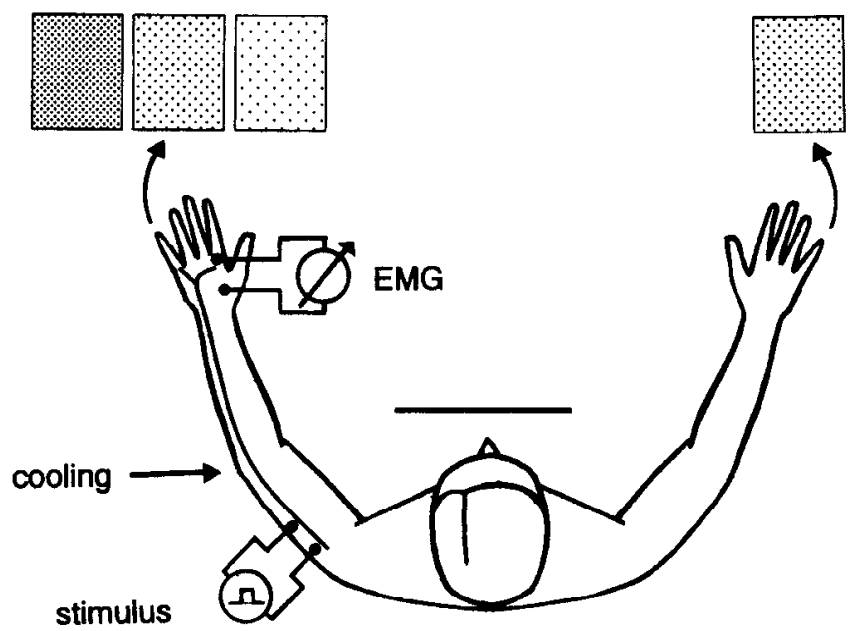

Figure 1. The experimental arrangement. The subject compared the roughness of surfaces embossed with dots by scanning them with the little fingers. The left elbow was cooled in order to interfere unilaterally with conduction in the ulnar nerve innervating the little finger. Test surfaces with different dot spacings were presented to the cooled side for comparison with a standard surface presented to the other side. The effects of cooling on axonal conduction were monitored by stimulating the ulnar nerve in the upper arm and recording the EMG from the FDI, which the ulnar nerve supplies.

as two-dimensional spatial event plots (SEPs), as described by Phillips et al. (1992). The impulse sequence evoked by each scan was plotted as a row of dots; each dot corresponded to a spike, with its position corresponding to the longitudinal position of the dot array at the time of occurrence of the spike. The row produced by each successive scan was plotted $0.2 \mathrm{~mm}$ below its predecessor, corresponding to the concomitant shift of the dot array. (See Fig. 7 for typical examples of SEPs together with further explanation.) In spite of the differences of detail in the stimulating arrangements, the receptors can be presumed to have responded very similarly during our testing of roughness.

Modeling refractory effects (filtering). The effects of refractoriness were modeled by removing all spikes from the original spike trains that fell within a prolonged absolute refractory period of the previous spike. This was done with a simple computational filter that simulated an absolute refractory period (ARP) in the range of $1-10 \mathrm{msec}$ and that behaved in one of two ways: the "mild" filter allowed an impulse to pass through the cooled region as soon as the ARP following the last successful impulse (i.e., one that passed through) had expired; the "harsh" filter allowed an impulse to pass only if the preceding interspike interval in the original recording exceeded the ARP of the filter. In effect, the mild filter ignored impulses that failed to pass, but that in reality might have left the nerve in a refractory state. In contrast, the harsh filter assumed that all impulses, whether they passed or not, would leave the nerve in a state of absolute refractoriness for the defined period. Thus, the mild filter underestimates and the harsh filter overestimates the ability of a length of cooled nerve with a defined ARP to block impulscs. Both, however, are too simple to deal with the full complexities of the real situation in which, inter alia, the refractory period can increase with the duration of the spike train (Paintal, 1965b; Franz and Iggo, 1968).

Computation of spatial variation in SEPS. The spatial variation of impulse rate in SEPs was calculated in the way described by Connor et al. (1990). Each SEP was converted into a two-dimensional rate histogram, with a bin width of $0.1 \mathrm{~mm}$ in the $\mathrm{x}$ - (scanning) direction and $0.2 \mathrm{~mm}$ in the $y$-direction (the size of the step between successive scans). The instantaneous rate (impulses/sec) in each bin was determined by summing the number of whole and fractions of interspike intervals falling within the bin and dividing by the dwell time in the bin; with this algorithm, bins lying between a pair of spikes are allocated the frequency corresponding to the reciprocal of their interspike interval. This determination of instantaneous frequency can underestimate spatial patterning when individual dots elicit only a single spike on each 


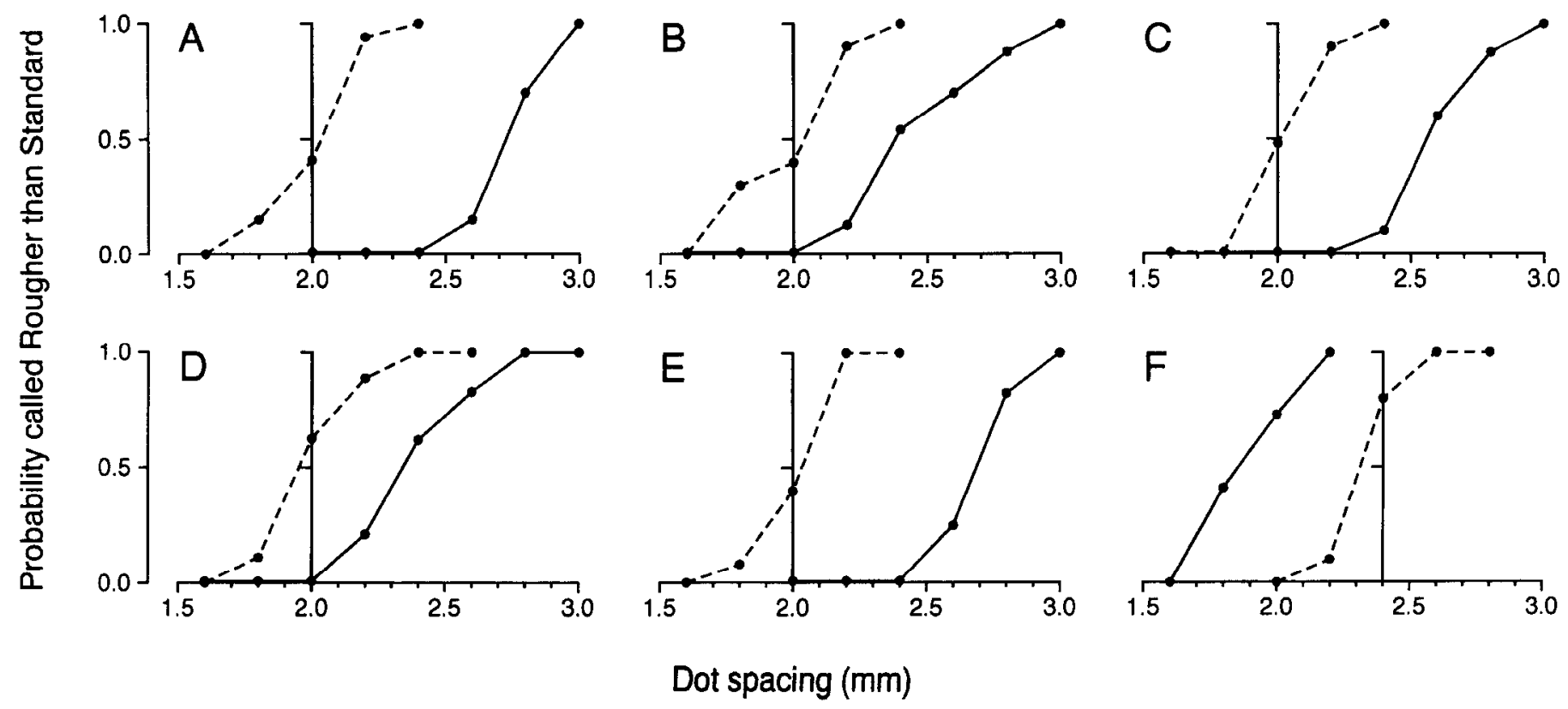

Figure 2. Effect of nerve cooling on perceived roughness of embossed dot arrays. Dots were of constant size but had variable spacing. $A-D$, Results from four subjects with the arrangement shown in Figure 1; cooling shifted the response curve to the right (dashed curve, both arms warm; solid curve, test arm cold). Thus, cooling made the surfaces feel smoother. Abscissa, dot spacing of test surfaces presented to the cooled side; spacing of the standard surface is marked by the vertical line $(2.0 \mathrm{~mm}$ for $A-E)$. Ordinate, probability that the test surface was reported as rougher than the standard surface. $E$, Same finding with greater than normal length of arm cooled $(35 \mathrm{~cm}$ instead of $10 \mathrm{~cm}$ used in $A-D) . F$, Test and standard surfaces reversed; $2.4 \mathrm{~mm}$ standard presented on cooled side; curve shifts to the left; see Results. $A, E$, and $F$ are from same subject.

scan, but this was not so in most of the present data (see Fig. 7); the situation would not have been improved by estimating frequency from the probability density of the spikes in each bin, since this destroys the information contained in the frequency code (cf. Matthews and Stein, 1969). The local spatial variation in the transformed SEP was determined by computing the mean absolute difference in firing rate between pairs of bins separated by $2 \mathrm{~mm} ; 2 \mathrm{~mm}$ was the optimal separation reported by Connor et al. (1990), but varying this value between 1 and $3 \mathrm{~mm}$ had little effect on the present findings. Thus,

spatial variation

$$
=\frac{1}{12} \sum_{a=15}^{180} \frac{1}{n x \cdot n y} \sum_{x=1}^{n x} \sum_{y=1}^{n y}|R(x+d x, y+d y)-R(x, y)|
$$

where $a$ is the angle between the bin pairs (varied between $15^{\circ}$ and $180^{\circ}$ ), $n x$ and $n y$ represent the number of bin pairs in the analysis patch, $d$ is the bin separation $(2 \mathrm{~mm}), d x=d \cdot \cos (a)$ and $d y=d \cdot \sin (a)$ are the $\mathrm{x}, \mathrm{y}$ displacements between the reference and comparison bins, and $R(x, y)$ represents the rate in the bin at spatial location $x, y$. The patches analyzed were $10 \mathrm{~mm}$ wide and $14 \mathrm{~mm}$ deep, as in the SEPs seen in Figure 7.

Short-term temporal variation in the SEP. For simplicity, temporal variation was assessed using the algorithm for spatial variation and restricting the analysis to the direction of scanning for which distance and time are interconvertible. Expressed in terms of time, the bin width of the temporal histogram derived from the SEP was $1.5 \mathrm{msec}$. A distance of $2 \mathrm{~mm}$ was again used, corresponding to $30 \mathrm{msec}$ at the scanning speeds used. The precise values varied slightly from afferent to afferent, because of small differences in scanning velocity. The same overall findings were obtained on varying the sampling interval between 15 and $45 \mathrm{msec}$.

\section{Results}

\section{Roughness of dot patterns with variable spacing}

Figure 2 shows six examples of the effect of cooling the arm on the perception of the roughness of surfaces consisting of embossed dots of constant size but different spacing. The hand remained warm throughout, so the effects are due to the effect of cooling on nervous conduction rather than to abnormalities in the generation of the sensory input by the receptors. When both arms were warm (dashed lines), the perception of roughness on the two sides was very similar. Arrays with a dot spacing of $1.8 \mathrm{~mm}$ or less were regularly reported as smoother than the 2 $\mathrm{mm}$ standard, and those with a spacing of $2.2 \mathrm{~mm}$ or greater were felt to be rougher. When identical $2 \mathrm{~mm}$ arrays were presented bilaterally, the probability of the test array being called rougher was about 0.5 , but the position of the curve as a whole is of greater significance. Occasionally there was a small bias of the curve to one side or the other.

When the arm was cooled, arrays with the standard spacing of $2 \mathrm{~mm}$ were unhesitatingly reported as feeling smoother than the identical array on the warm side and the dot spacing had to be increased appreciably before they felt equally rough, as shown by the solid lines in Figure 2. Plots $A-D$ show results for four subjects studied with cooling confined to the elbow; this shifted the curves to the right by $0.4-0.7 \mathrm{~mm}$. Without cooling, dot arrays with such an increase in spacing of 20-30\% feel much rougher than the standard. Plot $E$ shows a similar shift to plot $A$ on cooling a much greater length of arm of the same subject, suggesting that the effect arises primarily from the intensity of cooling rather than the length of nerve affected (see below). In $F$ the curve shifts to the left rather than the right because, as a control, the cooled side rather than the warm side was presented with the standard (now $2.4 \mathrm{~mm}$ ); the abscissa shows the dot spacing on the warm side that was required to match it, which decreased when cooling made the standard feel smoother.

The curves were restored to their normal positions after rewarming the arm, with the tubing still in place, confirming that the shifts were due to the cooling per se rather than to adventitious factors. For example, very slight edema of the hand occasionally occurred and could conceivably have altered the mechanical properties of the skin. When dot arrays with widely spaced dots were compared with the standard, with introspec- 
tion, subjects were able to distinguish between the percept of roughness and the percept of dot spacing. One subject volunteered the comment that arrays with widely spaced dots felt smooth during cooling because "the tops of the dots were rounded off."

\section{Within-hand control observations}

Figure $3 B$ provides further evidence that the sensory effects were due to cooling of nerves in the arm, rather than to some undefined local change in the hand, such as edema. As usual, the perceived roughness of a dot array presented to the little finger of the cooled arm was compared with a standard, but now the standard was presented to the index finger on the cooled side rather than the little finger on the warm side. The index finger is supplied by the median nerve, which runs deeply in the arm and so is much less affected by cooling than the ulnar nerve that supplies the little finger (Matthews, 1989). Thus, cooling should still reduce the roughness of the surface presented to the little finger relative to that of the standard, even though the standard is presented to the same hand. Figure $3 B$ was obtained in this way and shows the usual shift to the right with cooling, confirming that the effect was not due to changes within the hand as a whole. However, the amount of shift was slightly less than that found in the same experiment with the normal arrangement (Fig. $3 A$ ). Figure $3 B$ shows incidentally that while the arm was warm, a surface felt rougher to the little finger than to the index finger of the same hand (dashed curve no longer symmetrical about $2.0 \mathrm{~mm}$ ); this is probably due to differences in the thickness of the skin at the two sites. Similar results were obtained on another occasion on the same subject.

Figure $3 C$ was obtained in the same experiment and shows that the cold-induced change in sensibility of the little finger was not accompanied by a correspondingly gross change in its discriminatory capacity. It might have been suggested that cooling made all the more closely spaced dot arrays feel the same. The subject's task was now to compare two surfaces that were both presented to the little finger of the cooled side and to report simply whether they felt the same or different; the standard had the usual $2 \mathrm{~mm}$ dot spacing, the test spacing was $2 \mathrm{~mm}$ or above, and the subject knew this. In spite of superficial similarities, such testing of discrimination is quite distinct from the earlier comparisons of roughness. During cooling (solid curve, Fig. $3 C$ ), dot arrays of 2.0 and $2.2 \mathrm{~mm}$ spacing were still reliably distinguished $(p=1.0)$ when presented in succession to the little finger at a time when both felt smoother than normal (Fig. 3A). Nor was any significant change in sensory acuity detected from the normal (dashed line). Due to time constraints the absolute limit of discrimination was not determined (cf. Lamb, 1983), but on present showing any small change could not have been responsible for the present changes in the perception of roughness. Similar results were obtained in two further experiments on the same subject, and in an experiment on a another subject (in two of the four experiments, a long length of arm was cooled and in two just the elbow).

\section{Roughness of gratings with constant spacing}

For the analysis of underlying mechanism there are certain advantages in studying surfaces in which roughness varies with the wavelength of a repeating structure (see Discussion), but it seemed desirable to establish that the conclusions held true for the simpler situation in which the spatial frequency remained constant. In initial experiments, on two subjects, the height of
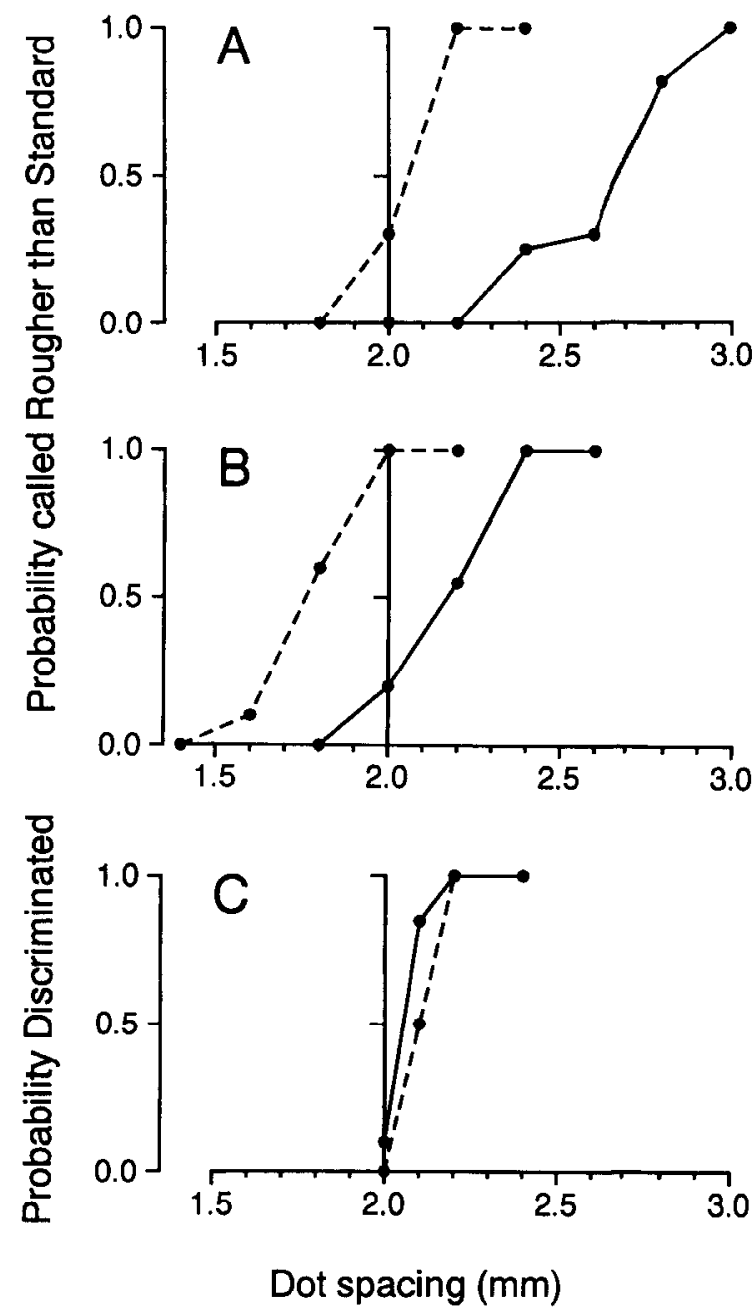

Figure 3. Within-hand control observations. All data illustrated were obtained from the same subject on the same occasion. $A$, Confirmation of effect with usual arrangement, as in Figure $2 A-E$. $B$, Effect of presenting the $2.0 \mathrm{~mm}$ standard to the index finger and the test surfaces to the little finger, both on the cooled side. $C$, Test of the discriminative ability of the little finger during cooling; standard and test surfaces were presented in turn to the little finger of the cooled side and the subject reported whether they were the same or different; see Results. Dashed lines, warm; solid lines, cold; $35 \mathrm{~cm}$ of arm cooled as in Figure $2 E$.

the dots was varied while keeping their spacing constant. The two major findings were then confirmed, namely, that a given surface felt smoother on the cooled side and that a dot array that normally felt rougher than a standard (on account of its greater dot height) came to feel smoother during cooling. However, limitations in the fabrication of the surfaces discouraged us from studying dot height more quantitatively.

Attention was thus switched to square-wave gratings, which have also been studied before (e.g., by Sathian et al., 1989). They provide a surface whose roughness can be varied controllably by altering the width of their grooves while keeping their height, edge profile, and spatial period constant. Figure 4 illustrates the effect of cooling on the perceived roughness of gratings, studied in the usual way (see Fig. 1) on three subjects. The findings replicate those obtained with dot arrays. Under normal conditions, in the range studied, the gratings form a series in which roughness increases monotonically with groove width (dashed lines). On cooling, the relation is shifted to the right (solid lines), showing that gratings that normally felt rough- 

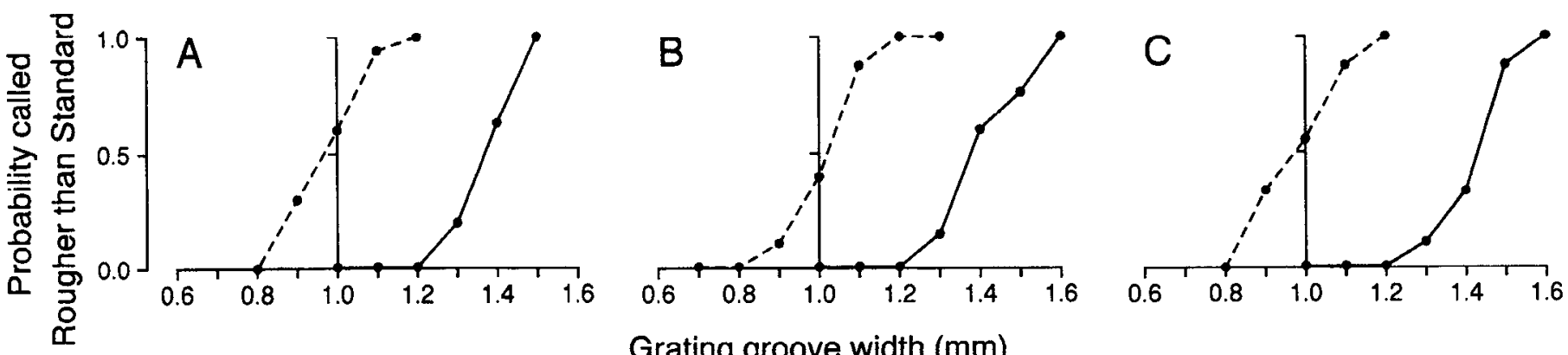

Grating groove width ( $\mathrm{mm}$ )

Figure 4. Effect of nerve cooling on perceived roughness of gratings. Test surfaces were presented to the cooled side and a standard to the other side: results for three subjects $(A-C)$ using gratings with a period of $2.0 \mathrm{~mm}$ but varying groove width (groove $1.0 \mathrm{~mm}$ wide for standard). The roughness of the gratings increased with their groove width. Cooling made the test gratings feel smoother than normal, shifting the response curve to the right. Dashed curves, test arm warm; solid curves, elbow cooled.

er than the standard came to feel smoother during cooling. Moreover, there was no overlap at all between the curves obtained in the presence and absence of cooling.

\section{Monitoring nerve conduction}

During each psychophysical experiment the action of cooling on conduction in the ulnar nerve was tested regularly by stimulating it in the upper arm, above the cooled region, and recording the EMG of the FDI (occasionally the nerve was stimulated at the wrist and the F-wave studied; see Materials and Methods). The ulnar nerve supplies both this muscle and the skin of the little finger. The larger sensory axons are the same size as motor axons, and in the cat are similarly affected by cooling (Paintal, 1965a). Also, our observations on motor fibers are in accord with measurements on human sensory fibers studied over a more limited range of cooling (e.g., Buchtal and Rosenflack, 1966; Lowitzsch et al., 1977).

The first concern is whether the cooling produced a total nerve block. This seems unlikely as the temperature was too high, allowing for the fact that the nerve trunks will have been warmer than the skin, which remained above $10^{\circ} \mathrm{C}$ (Bickford, 1939; Paintal, 1965a). More directly, a large EMG continued to be elicited by a single shock as illustrated in Figure 5; these recordings were made shortly after demonstrating the usual changes in roughness. Figure $5 A$ shows that during cooling the response was delayed by $2 \mathrm{msec}$, due to a slowing of conduction in the cooled region. However, its size and waveform were largely unaffected, as emphasized by Figure $5 B$, in which the recordings are superimposed. A large EMG invariably persisted with the present mode of cooling, although in not all experiments did it correspond so precisely with that obtained when the arm was warm, but tended to be smaller. This was partly due to unrelated changes in the recording conditions, since the EMG then usually failed to return to its original size on rewarming. Moreover, cooling can reduce the size of the EMG without blocking conduction, because it slows conduction and so desynchronizes the unitary diphasic potentials contributing to the EMG. Thus, the recordings cannot exclude a small degree of nerve block having occurred on occasion, but experiments like that of Figure 5 exclude it from being uniquely responsible for the observed psychophysical effects.

\section{Monitoring refractory period}

Cooling invariably interfered with the transmission of trains of impulses at relatively high frequency, as is well known from animal work (Paintal, 1965b; Franz and Iggo, 1968). Cooling prolongs the refractory period of nerve so that any impulse following its predecessor too closely is blocked; moreover, a blocked impulse still produces some refractoriness, so that its successor may also be blocked. Stimulation of the ulnar nerve with pairs of shocks showed that at the time of the perceptual changes the effect was large enough to block the higher-frequency components of the afferent discharge elicited by the present stimuli. When the arm was warm, two stimuli separated by 2$3 \mathrm{mscc}$ regularly cvoked two separate responses in FDI. When the arm was cooled, the stimulus interval had to be increased to $5-10 \mathrm{msec}$ before the second impulse was transmitted to the muscle.

Figure $5 C$ shows an example of such refractory block of a second impulse following $7 \mathrm{msec}$ after the first. The completeness of the block is shown by superimposing the response upon that to a single stimulus; the two traces are effectively identical. The superimposed recordings in Figure $5 D$ show the progressive recovery of the second response on increasing the stimulus interval from 10 to $30 \mathrm{msec}$. However, the size of the second potential does not provide a 1:1 measure of the number of motor axons transmitting a second impulse, because of a variable dispersion of its subcomponents. Nonetheless, an appreciable number of axons would seem to be failing at an interval of $10 \mathrm{msec}$, suggesting that discharges at $100 \mathrm{~Hz}$ would fail to be transmitted normally. For comparison, single-unit recording in human has, shown that both rapidly and slowly adapting receptors may fire at up to $300 \mathrm{~Hz}$ on scanning the finger over the present type of dot array (Phillips et al., 1992).

During the relative refractory period the conduction velocity of an axon is reduced below its normal value; the effect becomes particularly marked when the refractory period is prolonged by cooling. This has the interesting consequence that the interval between a pair of spikes is increased during their successful transmission along a cooled region of nerve. The effect seems large enough to have contributed to the present cold-induced distortion of afferent signaling. In Figure $5 D$ the earliest of the action potentials evoked by the second stimulus occurred 13.3 msec rather than $10 \mathrm{msec}$ (the stimulus interval) after the first action potential; its instantaneous frequency had thus been reduced from $100 / \mathrm{sec}$ to $75 / \mathrm{sec}$. Physiologically evoked afferent discharges will have been similarly affected, with a scaling down of the frequency of discharge at the beginning of a burst even when impulses were not actually blocked. Moreover, because of this effect, testing with just two stimuli tends to underestimate 

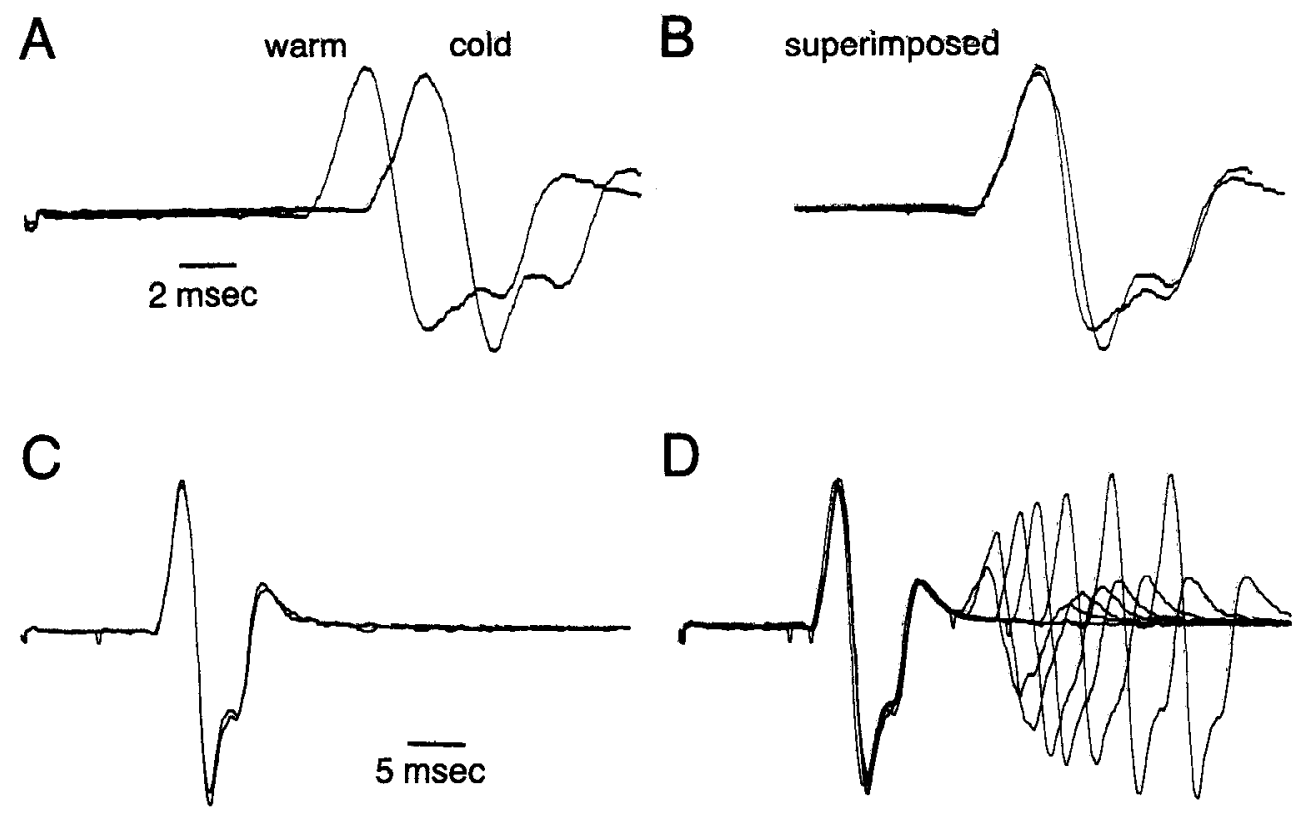

Figure 5. Monitoring nerve condurction during cooling. The EMG from the FDI was recorded on stimulating the ulnar nerve above the cooled region. The recordings were made shorty after demonstrating the reduction in the perception of roughness shown in Figure $2 B$. $A$, The responses to single shocks before and during cooling at the elbow; note increase in latency. $B$, The same responses aligned laterally to improve comparison. $C$, The response during cooling evoked by two stimuli separated by $7 \mathrm{msec}$ (note stimulus artifacts) superimposed upon the response to a single stimulus. They are identical, indicating that the nerve volley elicited by the second stimulus was blocked in the cooled region due to a greatly prolonged absolute refractory period (normal valuc below $2 \mathrm{msec}$ ). $D$, Duration of refractoriness shown by superimposing the responses to pairs of stimuli of increasing separation $(10,12,15,17$, $20,25,30 \mathrm{msec})$. the extent of high-frequency block; in a discharge at constant frequency the second impulse may be able to pass through a cooled region when all subsequent spikes are blocked (Paintal, 1965b; Franz and Iggo, 1968).

\section{Significance of temporal dispersion}

Cooling produces a dramatic slowing of nervous conduction, which can be reduced to $5 \%$ of its normal value before block occurs. If all afferents were the same size this would be largely immaterial in the present context, since it would merely slightly delay the time at which the sensory image was processed without changing its attributes. However, functionally equivalent afferents may have conduction velocities varying by up to a factor of two, and it is well recognized that this will degrade the fine details of a neural image (Iohnson and Lamb, 1981). Such blurring must increase during cooling; the conduction velocity of an axon is then reduced by a fixed percentage of its initial value, accentuating the temporal dispersion between impulses that were initiated simultaneously in afferents of different size. This raises the question of whether temporal dispersion rather than refractoriness could have been responsible for the observed changes in the perception of roughness. However, varying the parameters of cooling showed that with the present arrangements any effects of dispersion were too small to have been responsible.

Temporal dispersion was produced without appreciable refractoriness by cooling a long length of the arm relatively mildly. This is possible because of the rather different dependence of these two properties of nerve on temperature. Conduction velocity is approximately linearly related to temperature; thus, the dispersion of the spikes in a population of axons will increase in direct proportion to the degree of cooling and the length of nerve affected. In contrast, the refractory period increases as some higher-order function of the degree of cooling and is only appreciably prolonged when the temperature has fallen to 10$15^{\circ} \mathrm{C}$ (Paintal, 1965b). Moreover, the extent of high-frequency block will depend upon the temperature of the coldest part of the nerve, and not upon summation along its length as with dispersion. Figure 6 contrasts the effects of the two modes of cooling on the perception of roughness. Figure $6 \mathrm{~A}$ shows the usual action of cooling at the elbow, which again produced about $2 \mathrm{msec}$ slowing of conduction, as in Figure $5 \mathrm{~A}$. Figure $6 \mathrm{~B}$ shows that less severe cooling of the whole arm of the same subject had no detectable effect on roughness perception, although it produced a slowing of $4 \mathrm{msec}$ and thus twice as much dispersion of the afferent signals. It follows that temporal dispersion cannot have contributed significantly to the action of the more severe local cool, confirming that it can be attributed to refractoriness. The same result was obtained on making the comparison in another subject. In this case the ARP was measured during the mild cooling and found to be below $2 \mathrm{msec}$, which would not have affected the normal afferent discharges.

In four cxpcriments, all on the same subject, the whole arm was cooled intensely, rather than just the elbow, so as to produce a larger effect on dispersion. Conduction along the cooled nerve then took some $10 \mathrm{msec}$ longer than normal, as opposed to the usual 2-3 msec with the restricted cooling. The effect of cooling on the perception of roughness was the same in the two cases (compare Fig. $2 A$ and $E$ ). The long cool would have increased sensory dispersion to about $50 \%$ above its normal value (conduction time from finger to spinal cord is approximately 20 $\mathrm{msec}$ ). It is therefore unlikely that dispersion can have contributed anything significant to our findings.

\section{Discussion}

The basic finding is that when the ulnar nerve is cooled, a given surface feels smoother than normal to the touch. The surfaces studied were either raised dots of variable spacing or gratings with constant repeat spacing, providing a reasonable basis for generalization. The effect could not have been due to a total cold block of afferent nerve fibers; the motor fibers were not blocked, and medullated fibers all behave similarly (Paintal, 1965a; Franz and Iggo, 1968). Cooling did increase the normal temporal dispersion of the impulses traveling in different-sized afferents, but this was excluded as the causative factor. However, the effect can be satisfactorily attributed to cooling increasing the refractory period of the sensory axons leading to Wedensky inhibition of the high-frequency components of their discharge. The refractory period of the similar-diameter motor fibers was 


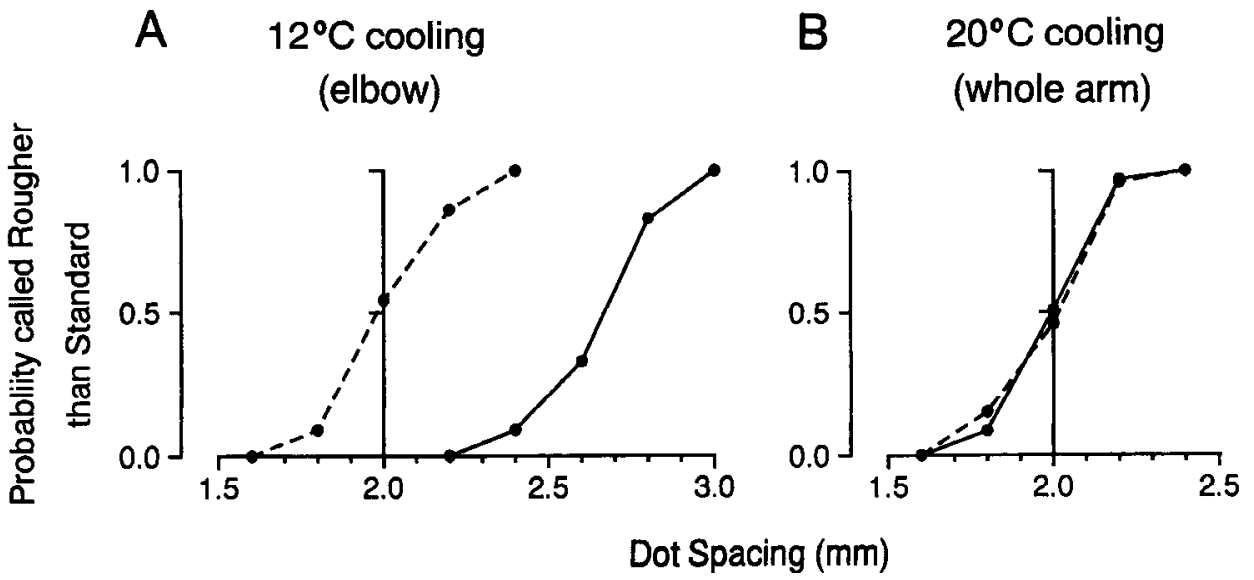

Figure 6. Effect of varying cooling conditions. $A$, Effect of cooling a short length of nerve (about $10 \mathrm{~cm}$ ) at the elbow (skin temperature, $12^{\circ} \mathrm{C}$ ); this slowed conduction by $2 \mathrm{msec}$ and produced a shift of the response curve to the right (as in Fig. 2). $B$, Effect of mildly cooling a longer length of nerve (about $35 \mathrm{~cm}$ ) extending above and below the elbow (skin temperature, $20^{\circ} \mathrm{C}$ ); this slowed conduction by $4 \mathrm{msec}$, but had no effect on the response curve (dashed line, warm; solid line, cold). Thus, mild cooling of a long length of nerve failed to change the perception of roughness even though it produced more slowing of conduction than severe cooling at the elbow. It follows that the altered perception in $A$ cannot be due to the effects of dispersion, since this would have been greater in $B$. Temperature was measured with a thermocouple between the skin and the coolant tubing. Slowing was determined using the F-wave in both $A$ and $B$; see Materials and Methods.

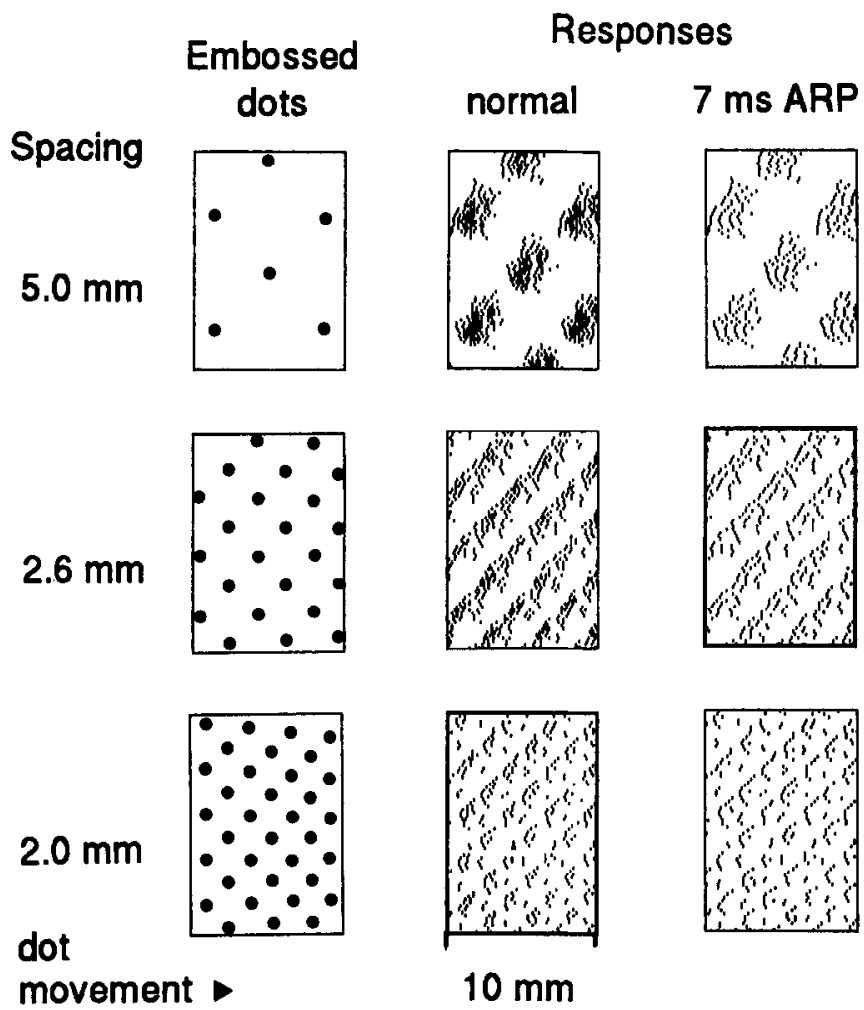

Figure 7. Effect of nerve refractorincss on the afferent discharge of a single human SAI unit. Results were computed from the data of Phillips et al. (1992). In the original experiments the dot patterns shown to the left were scanned repeatedly over the fingerpad, resulting in the SEPs shown in the middle column. Each cluster of dots within the SEP is analogous to a plot of the receptive field of the afferent, with each spike plotted as a point on the same $x-y$-coordinates as the stimulus dots; the dot array was moving from left to right at constant velocity $(69 \mathrm{~mm}$ / sec) so that the horizontal separation between the points with the same $y$-value gives the instantaneous frequency of firing. The SEPs in the right column were constructed by filtering the original spike trains to simulate an ARP of $7 \mathrm{msec}$; no spike was then left within $7 \mathrm{msec}$ of its predecessor ("mild" filtering). In the psychophysical experiments a 2.6 $\mathrm{mm}$ dot spacing presented to the cooled side was judged equal in roughness to the standard $(2 \mathrm{~mm})$ dot spacing presented to the unaffected side. The corresponding SEPs are indicated with heavy borders. increased sufficiently to block impulses at the frequencies at which the afferents have been shown to fire in human. In essence, as explained below, it appears that for a surface to feel rough to a finger, individual afferents must vary their frequency of firing widely as the finger is moved across the surface; eliminate the highest frequencies and the surface feels smoother, even though its main spatial characteristics may remain distinguishable.

\section{Modeling the effects of Wedensky inhibition}

The cutaneous afferent responses evoked by scanning an array of embossed dots over the finger pad were recently recorded in human by Phillips ct al. (1992). Using their data files we have computed the effects of prolonging the refractory period on the spatiotemporal patterning of the afferent discharges. Figure 7 shows samples of an original unitary recording from a slowly adapting (SAI) afferent (middle column) together with filtered recordings (right column) showing the effects of increasing the absolute refractory period of the afferent axon to $7 \mathrm{msec}$ and removing the "blocked" spikes (see Materials and Methods). The data are displayed as an SEP in which both axes show distance. Each point in the SEP corresponds to a spike, and each clump of spikes corresponds to the afferent representation of a dot on the surface being scanned. An SEP summarizes the responses to a number of scans, in each of which every dot produces a burst of spikes at frequencies up to $300 \mathrm{~Hz}$ as it is swept across the unit's receptive field; the instantaneous frequency of firing can be deduced from the spacing between successive spikes in the same scan. The SEP thus indicates the degree to which spatial details of the stimulus can be resolved in the neural image.

The top records in Figure 7 show the findings when the dots were more widely separated than those currently studied psychophysically; each dot then elicited a well-demarcated clump of spikes in the SEP, making it easier to see what was happening. On increasing the ARP to $7 \mathrm{msec}$, as found in the main experiments (see Fig. 5), each dot continued to produce a clump of spikes in the SEP, but the number of spikes and their frequency were markedly reduced. Thus, the main features of the spatio- 
temporal pattern were preserved, but the contrast between the neural image of the dot and its surround was greatly reduced. In this example spikes were deleted only when they followed within $7 \mathrm{msec}$ of a spike that had been successfully transmitted through the simulated cooled region ("mild" filtering). If blocked spikes were allowed to leave the axon refractory, as can happen in life (Paintal, 1965b), then the effects were similar in kind but appreciably more drastic ("harsh" filtering).

The remaining records in Figure 7 show the responses for the dot spacings currently tested psychophysically. The dot array can still be detected in the normal neural image, but the clump of spikes representing each dot has been reduced in all directions and is less well defined (due to interference from adjacent dots simultaneously falling within the receptive field of the afferent). However, making the nerve refractory has the same qualitative effects as with widely separated dots. Both the average number of spikes per dot and their instantaneous frequency are reduced, so the gross patterning is preserved while the spatiotemporal contrast is reduced; again, harsh filtering had a similar but quantitatively greater effect. In the psychophysical testing, $2.6 \mathrm{~mm}$ spacing on the cooled side was approximately equated with 2.0 $\mathrm{mm}$ on the normal side (Fig. 2). Likewise, the $2.6 \mathrm{~mm}$ filtered SEP in Figure 7 appears similar to the normal $2.0 \mathrm{~mm} \mathrm{SEP}$; both are shown with heavy borders in Figure 7 . Their quantitative similarities are demonstrated below.

Qualitatively similar results were obtained for all afferents with small receptive fields, whether slowly or rapidly adapting (15 SAI and 22 FAI afferents examined). The SEPs for largefield, fast-adapting afferents (FAII afferents) were complex, with little direct representation of the dot pattern. However, they fired at moderately high frequencies during scanning and, as with the type I receptors, appreciable numbers of spikes were eliminated on increasing the refractory period (eight FAII afferents examined). In contrast, slowly adapting afferents with large receptive fields (SAII afferents) were only weakly excited by the present stimuli and fired at such low mean frequencies (7-24 impulses/sec) that they were almost completely unaffected by increasing the refractory period by up to $10 \mathrm{msec}$ (this eliminated less than $3 \%$ of the spikes of the six SAII afferents studied).

\section{Codes for roughness}

Mean rate. The gross alteration in SAI, FAI, and FAII discharges with cooling must be individually or severally responsible for the observed changes in roughness. The question remains, for each receptor, as to precisely which of the alterations in its pattern of discharge are responsible for the effects, and which are adventitiously linked. The mean frequency of discharge is a potentially significant variable. It will fall with cooling, as the higher-frequency discharges are eliminated by refractoriness. Under normal conditions, the mean impulse frequency of individual SAI and FAII afferents regularly decreased on decreasing the dot spacing from 2.6 to $2.0 \mathrm{~mm}$. Thus, for these afferents, roughness is correlated with mean frequency in both circumstances. In contrast, half the FAI afferents increased rather than decreased their firing rate on decreasing the dot spacing in the same range. On studying a much wider range of spacing, Connor et al. (1990) rejected mean firing rate of all types of afferent as the relevant variable, since surfaces that evoked the same firing rate for monkey affercnts often cvoked very different judgements of roughness. Thus, the reduction of the mean firing frequency with cooling is unlikely to be responsible for the observed reduction of roughness.
Spatial variation. Connor et al. (1990) suggested that the percept of roughness depended on the variation in afferent firing rate, with the sensory centers compounding the information from a number of adjacent afferents. Using SEPs for monkey afferents, they calculated an index of spatial variation, with dimensions of impulses per second, which provided a measure of the gradients in firing across an array of afferent fibers, and which proved to be highly correlated with their psychophysical measurements of roughness. We have now computed this index for human data; the absolute values were remarkably similar to those for the monkey data.

Figure 8 shows the effect of increasing the refractory period on this index. For both SAI and FAI afferents (left) spatial variation decreases with simulated cooling; moreover, the two types of afferent behave identically. Of particular note is that the spatial variation for $2.6 \mathrm{~mm}$ spacing with a refractory period of 5-7 msec corresponds to the normal (warm) value for the 2.0 $\mathrm{mm}$ standard, in agreement with the psychophysical findings. The FAII afferents (right) behaved similarly (but see below). The SAII afferents were unaffected, in accordance with the failure of simulated cooling to alter their responses. More significantly, their normal SEPs contained very much less temporal or spatial variation than those of the other afferents. Thus, the SAII afferents appear incapable of making an effective contribution to the perception of roughness, and so are unlikely to be involved.

Temporal variation. The good agreement between modeling and experiment lends support to the idea that roughness is related to local spatial patterning. However, taken by itself, such a comparison is insufficient to prove that spatial aspects of the signal must be involved, since the temporal pattern of firing was also changed. This was examined by computing a related index of short-term temporal variation of firing, again expressed in impulses per second (see Materials and Methods); it represented the average difference in firing frequency between a pair of samples with a temporal separation of about $30 \mathrm{msec}$ (corresponding to $2 \mathrm{~mm}$ movement of the dot array). For the present human data, as with the earlier monkey data (Connor et al., 1990), the short-term temporal variation was larger with $2.6 \mathrm{~mm}$ than with $2.0 \mathrm{~mm}$ spacing. Rather naturally, the index decreased with simulated cooling, since the highest frequencies were removed. More significantly, as with the spatial index, the absolute value of the tcmporal index for a spacing of $2.6 \mathrm{~mm}$ during simulated cooling (ARP, 5-7 msec) was very similar to the normal value for $2.0 \mathrm{~mm}$ spacing. Thus, the present analysis does nothing to distinguish between spatial and temporal codes in the signaling of roughness, since simulated cooling reduced both of them by the appropriate amount. Nonetheless, the actual code seems likely to be a spatial one, because this automatically provides for the known constancy of roughness with scanning speed found when the subject makes the scanning movements (Lederman, 1974). Furthermore, the recent findings by Connor and Johnson (1992) fit the predictions of a spatial, but not a temporal, coding hypothesis.

FAII afferents. In the present analysis, both SAI and FAI afferents have the potential to be involved in spatial coding, and seem likely to be; their response to dot scanning is remarkably similar, both before and during simulated cooling. However, in spite of the quantitative similaritics, the FAII afferents are unlikely to contribute to spatial signaling. In their case, the SEP is potentially misleading, since FAIIs have a low innervation density and large overlapping receptive fields ex- 

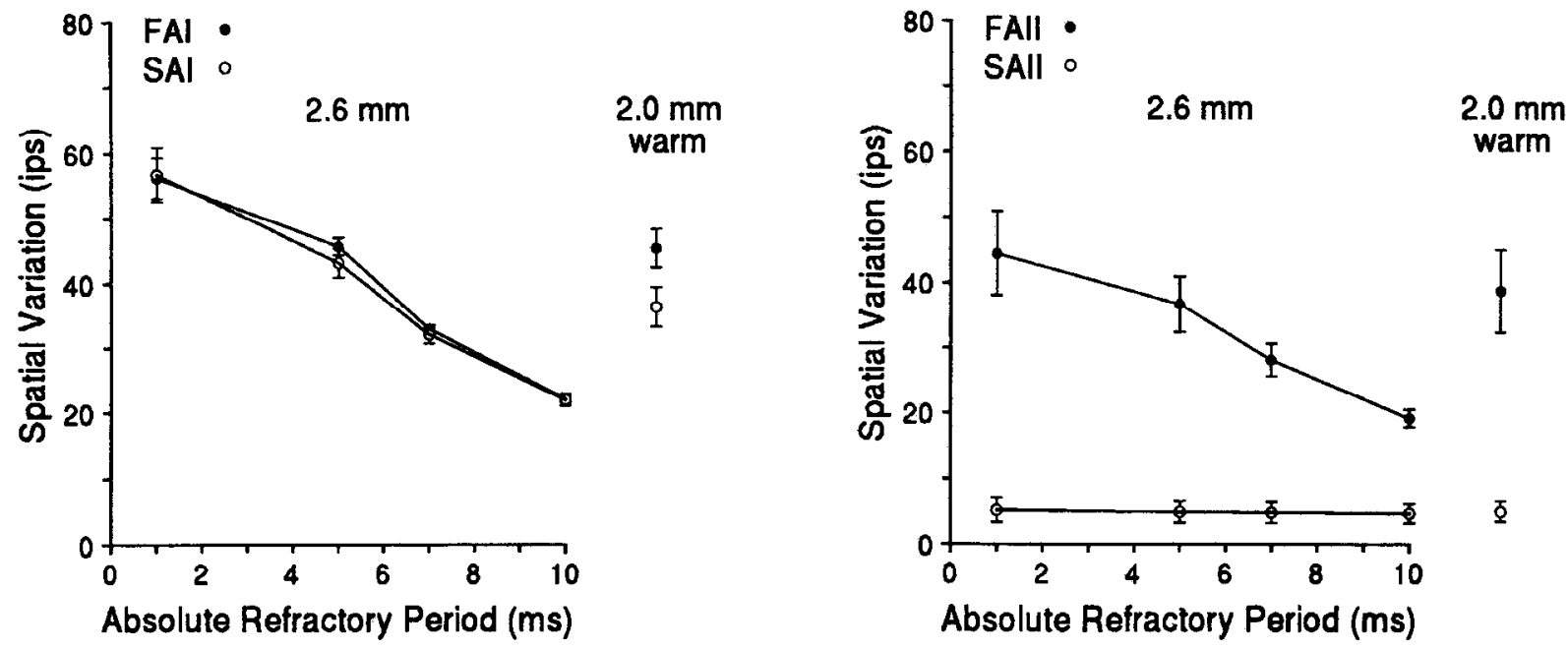

Figure 8. Effect of refractoriness on index of spatial variation. The index (mean \pm SEM) was computed from the SEPs of the four types of mechanoreceptive afferent. Left, type I receptors (open circles, mean of $15 \mathrm{SAI}$; solid circles, mean of $22 \mathrm{FAI}$ ); right, type II receptors (open circles, mean of 6 SAII; solid circles, mean of 8 FAII). This index averages the two-dimensional spatial gradients of firing in the SEP; the larger the index, the greater the spatial contrast. The joined points show the progressive reduction in the value of the index for $2.6 \mathrm{~mm}$ spacing on increasing the absolute refractory period ("mild" filtering). The individual points to the right of each panel show the normal value with $2.0 \mathrm{~mm}$ spacing. The spatial gradient was calculated across a distance of $2 \mathrm{~mm}$; very similar results were obtained using $1 \mathrm{~mm}$ or $3 \mathrm{~mm}$.

tending far outside the area stimulated: they originate from Pacinian corpuscles. For type I receptors with their small receptive fields and high innervation density the patterning in the SEP will approximately represent the spatial pattern of activity that the CNS receives from an array of receptors; the present analysis of the SEP could thus potentially be performed by synaptic circuitry. But the CNS seems unlikely to be organized to extract spatial detail from the FAII messages with similar precision. Moreover, with their exquisite sensitivity, the FAII receptors may well be responding to microvibrations set up by spatial irregularitics in the skin rather than to the precise location of the dot within their receptive field. The patterning in the SEP would then be similar for a number of adjacent FAII receptors, with overlapping fields, and contain no useful spatial information. Thus, FAII afferents are unlikely to contribute to a spatial analysis of roughness. However, temporal coding is another matter and Johnson and Hsiao (1992) recently noted that FAII afferents could well be responsible for the perception of the roughness of finely grained surfaces on the basis of a temporal code. Our calculations confirm that short-term temporal variation in FAII firing has the potential to contribute to roughness perception, since like spatial variation it was decreased by simulated cooling. Temporal variation should be readily determinable by the CNS from an array of receptors with large receptive fields, especially if the variations in their firing rate should be correlated.

Gratings. The reduction in perceived roughness of gratings with cooling can be explained in much the same way as for dot arrays. Their excitatory action has been widely studied in the monkey and it has been shown that excitation occurs at the leading edge of the bars. Both the number of impulses and their instantaneous frequency tend to increase on widening the grooves while holding the spatial frequency constant (Goodwin et al., 1989). As with the dots, the increase of refractory period with cooling would thin out these bursts and reduce the spatiotemporal contrast of the neural image. The same would be expected for other types of surface.

\section{Preservation of sensory function}

In spite of the presumed large changes in afferent firing patterns, the overall sense of texture was in some ways remarkably robust during cooling. The dot arrays still felt rough, and careful testing was required to demonstrate the perceptual changes. Rather similarly, there may be no overt sensory symptoms in the early stages of diabetic neuropathy, in spite of the failure of a number of receptors to discharge repetitively (Mackel, 1989). Such preservation of function is presumably related to the continued ability of the affected afferents to signal differences between pairs of surfaces, in our case when presented sequentially on the cooled side. This finds a ready explanation in the preservation of the gross structure in the SEP. Each dot continued to evoke a recognizable response, so the potential to discriminate dot spacing remains. It may be concluded that Wedensky inhibition of afferent firing provides a satisfactory explanation for the action of cooling in reducing roughness without gross sensory derangement.

\section{References}

Bickford RG (1939) The fibre dissociation produced by cooling human nerves. Clin Sci 4:159-165.

Buchtal F, Rosenflack A (1966) Evoked action potentials and conduction velocity in human sensory nerves. Brain Res 3:1-122.

Connor CE, Johnson KO (1992) Neural coding of tactile texture: comparison of spatial and temporal mechanisms for roughness perception. J Neurosci 12:3414-3426.

Connor CE, Hsiao SS, Phillips JR, Johnson KO (1990) Tactile roughness: neural codes that account for psychophysical magnitude estimates. J Neurosci 10:3823-3836.

Douglas WW, Malcolm JL (1955) The effect of localised cooling on conduction in cat nerves. J Physiol (Lond) 130:53-71.

Franz DN, Iggo A (1968) Conduction failure in myelinated and nonmyelinated axons at low temperatures. J Physiol (Lond) 199:319345.

Fulton JF (1955) A textbook of physiology, 17th ed. Philadelphia: Saunders.

Goodwin AW, John KT, Sathian K, Darian-Smith I (1989) Spatial and temporal factors determining afferent fiber responses to a grating 
moving sinusoidally over the monkey's finger pad. J Neurosci 9:12801293.

Johnson KO, Hsiao SS (1992) Neural mechanisms of tactual form and texture presentation. Annu Rev Neurosci 15:227-250.

Johnson KO, Lamb GD (1981) Neural mechanisms of spatial tactile discrimination: neural patterns evoked by Braille-like dot patterns in the monkey. J Physiol (Lond) 310:117-144.

Lamb GD (1983) Tactile discrimination of textured surfaces: psychophysical performance measurements in humans. J Physiol (Lond) 338: $551-565$.

Lederman SJ (1974) Tactile roughness of grooved surfaces: the touching process and effects of macro- and microsurface structure. Percept Psychophys 16:385-395.

Lowitzsch K, Hopf HC, Galland J (1977) Changes of sensory conduction velocity and refractory periods with decreasing tissue temperature in man. $J$ Neurol 216:181-188.

Mackel R (1989) Properties of cutaneous afferents in diabetic neuropathy. Brain 112:1359-1376.

Matthews PBC (1989) Long-latency stretch reflexes of two intrinsic muscles of the human hand analyzed by cooling the arm. J Physiol (Lond) 419:519-538.

Matthews PBC, Phillips JR (1990) Effects of cooling human nerves on the perception of roughness. J Physiol (Lond) 429:28P.
Matthews PBC, Stein RB (1969) The sensitivity of muscle spindle afferents to small sinusoidal changes in length. J Physiol (Lond) 200: 723-743.

Mountcastle VB, LaMotte RH, Carli G (1972) Detection thresholds for stimuli in humans and monkeys: comparison with threshold events in mechanoreceptive afferent nerve fibers innervating the monkey hand. J Neurophysiol 35:122-136.

Paintal AS (1965a) Block of conduction in mammalian myelinated nerve fibres by low temperatures. J Physiol (Lond) 180:1-19.

Paintal AS (1965b) Effects of temperature on conduction in single vagal and saphenous myelinated nerve fibres of the cat. J Physiol (Lond) 180:20-49.

Phillips JR, Johansson RS, Johnson KO (1992) Responses of human mechanoreceptive afferents to embossed dot arrays scanned across the fingerpad. J Neurosci 12:827-839.

Sathian K, Goodwin AW, John KT, Darian-Smith I (1989) Perceived roughness of a grating: correlation with responses of mechanoreceptive afferents innervating the monkey's finger pad. J Neurosci 9:12731279.

Sinclair DC, Hinshaw JR (1951) Sensory changes in nerve block induced by cooling. Brain 74:318-335. 